\title{
SEPARABLE CRITERIA FOR $G$-DIAGRAMS OVER COMMUTATIVE RINGS
}

\author{
CHARLES WINFRED ROARK
}

\begin{abstract}
Let $S$ be a commutative, separable algebra over the commutative ring $R$ and finitely generated and projective as an $R$-module. Suppose $G$ is a group of ring automorphisms of $S$ stabilizing $R$ setwise. It is shown that for the ring of invariants $S^{G}$ to be a strongly separable extension of $R^{G}$ it is necessary that $R \cdot S^{G}$ be $R$-separable; and it is shown that this condition is sufficient when $R$ and $S$ are finitely generated algebras over an algebraically closed field and $G$ is a linearly reductive algebraic group acting rationally on S.
\end{abstract}

Recall that $S$ is a strongly separable $R$-algebra if $S$ is a separable $R$-algebra and a finitely generated and projective $R$-module [DI]. We say that $S$ is a Galois extension of $R$ with finite group $\Gamma$ of $R$-algebra automorphisms of $S$ if it satisfies the Chase-Harrison-Rosenberg definition of a Galois extension [DI]: $S^{\Gamma}=R$, and $l: S \otimes_{R} S \rightarrow C(\Gamma, S)$ is an isomorphism, where $l(s \otimes t)(\gamma)$ $=s \gamma(t)$ and $C(\Gamma, S)$ is all functions from $\Gamma$ to $S$. It is important for our purposes to note that if $l$ is an epimorphism then $l$ is an isomorphism.

Definition. Let $G$ be a subgroup of the ring automorphisms of $S$ such that $G$ restricted to $R$ is contained in the ring automorphisms of $R$. Then we have the following diagram of inclusions:

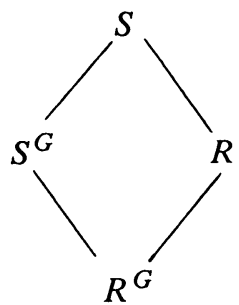

Such a diagram is called a $G$-diagram of $S$ over $R$.

This paper is concerned with the following question: If we have a $G$ diagram of $S$ over $R$ with $S$ a strongly separable (Galois) $R$-algebra, is $S^{G}$ a strongly separable (Galois) $R^{G}$-algebra? We begin by finding necessary conditions.

Received by the editors May 28, 1976.

AMS (MOS) subject classifications (1970). Primary 16Al6, 20 G99.

Key words and phrases. Separable algebra, Galois extension, linearly reductive algebraic group, rational $G$-module, $G$-ergodic. 
Proposition 1. Suppose we have a $G$-diagram of $S$ over $R$.

(a) If $S^{G}$ is a separable $R^{G}$-separable, then $R \cdot S^{G}$ is a separable $R$-algebra.

(b) Assume there is a finite group $\Gamma$ contained in the ring automorphisms of $S$ with $S^{\Gamma}=R$ and $\Gamma \mid S^{G}$ is contained in the ring automorphisms of $S^{G}$. If $S^{G}$ is a Galois extension of $R^{G}$ with group $\Gamma \mid S^{G}$, then $R \cdot S^{G}$ is a Galois extension of $R$ with group $\Gamma \mid R \cdot S^{G}$.

Proof. (a) Since $S^{G}$ is a separable $R^{G}, R \otimes_{R^{G}} S^{G}$ is a separable $R$-algebra. Now $\mu: R \otimes_{R^{G}} S^{G} \rightarrow R \cdot S^{G}$, where $\mu(r \otimes s)=r s$, is an epimorphism. Hence, $R \cdot S^{G}$ is a separable $R$-algebra.

(b) Since $\Gamma \mid S^{G}$ is contained in the ring automorphisms of $S^{G}$ and $S^{\Gamma}=R$, then $\Gamma \mid R \cdot S^{G}$ is contained in the ring automorphisms of $R \cdot S^{G}$ and $\left(R \cdot S^{G}\right)^{\Gamma}$ $=R$. Hence, we need only show that $l:\left(R \cdot S^{G}\right) \otimes_{R}\left(R \cdot S^{G}\right) \rightarrow C\left(\Gamma \mid R \cdot S^{G}\right.$, $\left.R \cdot S^{G}\right)$ is surjective. Since $S^{G}$ is a Galois extension of $R^{G}$, then $R \otimes_{R^{G}} S^{G}$ is a Galois extension of $R$ with group $\Gamma \mid R \otimes_{R^{G}} S^{G}$. Hence, $l^{\prime}:\left(R \otimes S^{G}\right)$ $\otimes\left(R \otimes S^{G}\right) \rightarrow C\left(\Gamma \mid R \otimes S^{G}, R \otimes S^{G}\right)$ is an isomorphism. Observe that the range and domain of $l$ are epimorphic images of those of $l^{\prime}$, and it follows that $l$ is a surjection.

For the remainder of the paper we assume that $G$ is a linearly reductive algebraic group over the algebraically closed field $k$ acting rationally on the finitely generated $k$-algebras $S$ and $R$. This will be called the Reductive Case.

Let $M$ be a rational $G$-module. Recall that $M^{G}=\{m$ in $M \mid g(m)=m$ for all $g$ in $G$, and $M$ is $G$-ergodic if $M^{G}=(0)$. Let $M_{G}$ denote the unique maximal $G$-ergodic submodule of $M$. Then $M=M^{G} \oplus M_{G}$, and the projection $P_{M}: M^{G} \oplus M_{G} \rightarrow M^{G}$ is called the Reynold's operator of $M[\mathrm{~F}, 5.3, \mathrm{p}$. 156]. If $M$ is also an $R$-module, we call $M$ a compatible $G$ and $R^{G}$-module if $g(r m)=g(r) g(m)$ for all $r$ in $R, m$ in $M, g$ in $G$.

Lemma 2. Let $M$ be a compatible $G$ and $R^{G}$-module. Then $P_{M}: M^{G} \oplus M_{G}$ $\rightarrow M^{G}$ is an $R^{G}$-module homomorphism. In particular, $M^{G}$ and $M_{G}$ are $R^{G}$ modules.

Proof. [F, 5.4, p. 156].

Lemma 3. Suppose that we have a $G$-diagram of $S$ over $R$ in the Reductive Case.

(a) If there is a finite group $\Gamma$ of automorphisms of $S^{G}$ with $\left(S^{G}\right)^{\Gamma}=R^{G}$, then $S^{G}$ is a finite $R^{G}$-module.

(b) If $R \cdot S^{G}$ is a finite $R$-module, then $S^{G}$ is a finite $R^{G}$-module.

(c) If $R \cdot S^{G}$ is a finite and projective $R$-module, then $S^{G}$ is a finite and projective $R^{G}$-module.

(d) Assume that $S$ is a strongly separable $R$-algebra. If $S^{G}$ is a separable $R^{G}$ algebra, then $S^{G}$ is a strongly separable $R^{G}$-algebra.

Proof. (a) Since $S$ is a finitely generated $k$-algebra and $G$ is linearly reductive, $S^{G}$ is a finitely generated $k$-algebra [F, 5.9, p. 160]; and, hence, $S^{G}$ is a finitely generated $R^{G}$-algebra. Since $\Gamma$ is finite and $\left(S^{G}\right)^{\Gamma}=R^{G}$, the ring $S^{G}$ is integral over $R^{G}$. This implies that $S^{G}$ is a finite $R^{G}$-module. 
(b) Since $R \cdot S^{G}$ is a finite $R$-module, we have $R \cdot S^{G}=\sum_{i=1}^{n} R x_{i}$ where we may assume the $x_{i}$ are in $S^{G}$. Then each $s$ in $S^{G}$ can be written $s=\sum r_{i} x_{i}=\sum r_{i}^{\prime} x_{i}+\sum r_{i}^{\prime \prime} x_{i}=\sum r_{i}^{\prime} x_{i}$ with $r_{i}$ in $R, r_{i}=r_{i}^{\prime}+r_{i}^{\prime \prime}, r_{i}^{\prime}$ in $R^{G}$, and $r_{i}^{\prime \prime}$ in $R_{G}$. The last equality follows since $S$ is a compatible $G$ and $R^{G}$ module, by Lemma 2 , and since $S=S^{G} \oplus S_{G}$. Hence $S^{G}$ is generated as an $R^{G}$-module by $\left\{x_{1}, \ldots, x_{n}\right\}$.

(c) Suppose $R \cdot S^{G}$ is a finite and projective $R$-module. Since $R \cdot S^{G}$ $=\sum_{i=1}^{n} R x_{i}$ with $x_{i}$ in $S^{G}$, there is a dual basis $\left\{f_{i}, x_{i}\right\}$ of $R \cdot S^{G}$ over $R$. Let $\Pi^{\prime}: R=R^{G} \oplus R_{G} \rightarrow R^{G}$ and $\Pi^{\prime \prime}: R=R^{G} \oplus R_{G} \rightarrow R_{G}$ be projections, and let $f_{i}^{\prime}=\Pi_{i}^{\prime} f_{i}$ and $f^{\prime \prime}{ }_{i}=\Pi^{\prime \prime} f_{i}$. Assume $x$ is in $S^{G}$. Then $x=\sum f_{i}(x) x_{i}$ $=\sum f_{i}^{\prime}(x) x_{i}+\sum f_{i}^{\prime \prime}(x) x_{i}=\sum f_{i}^{\prime}(x) x_{i}$ by the compatibility of the $G$ and $R$ module structure of $R \cdot S^{G}$, Lemma 2 , since $\left(R \cdot S^{G}\right)=S^{G} \oplus\left(R \cdot S^{G}\right)_{G}$. Hence, $\left\{f_{i}^{\prime}, x_{i}\right\}$ is a dual basis for $S^{G}$ and $R^{G}$, that is, $S^{G}$ is a finite and projective $R^{G}$-module.

(d) Since $S^{G}$ is a separable $R^{G}$-algebra, we apply Proposition 1(a) to get that $R \cdot S^{G}$ is a separable $R$-algebra. But $R \cdot S^{G}$, being a separable subextension of the strongly separable extension $S$, implies that $R \cdot S^{G}$ is a strongly separable $R$-algebra; in particular, $R \cdot S^{G}$ is a finite and projective $R$-module. Apply (c).

Suppose that we have a $G$-diagram of $S$ over $R$ in the Reductive Case and $S$ is a strongly separable $R$-algebra. Since $G$ is linearly reductive, $R^{G} \hookrightarrow R$ and $S^{G} \hookrightarrow S$ are split monomorphisms of $R^{G}$ and $S^{G}$-modules, respectively. Since $S$ is a strongly separable $R$-algebra, the inclusion $R \hookrightarrow S$ splits as an $R$ module. Recall that: If $T$ is any commutative $R^{G}$-algebra, then $G$ operates by $T$-algebra automorphism on $R \otimes_{R^{G}} T$ and the action is rational. Moreover $\left(R \otimes_{R^{G}} T\right)^{G}=T$ [F, 5.6, p. 157]. Let $T$ be an $R$-algebra. Hence, if we have a $G$-diagram

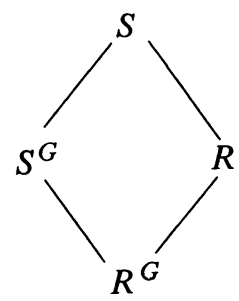

with $S$ a strongly separable $R$-algebra, then we may "tensor" to get a new $G$ diagram:

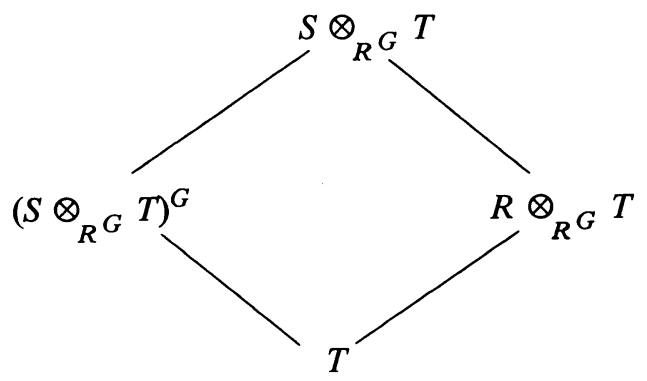


THEOREM 4. Suppose that we have a G-diagram of $S$ over $R$ in the Reductive Case with $S$ a strongly separable $R$-algebra. Then $S^{G}$ is a strongly separable $R^{G}$ algebra if and only if $S$ is a projective $R \cdot S^{G}$-module.

Proof. If $S^{G}$ is a strongly separable $R^{G}$-algebra, apply Proposition 1(a).

Conversely, assume that $S$ is a projective $R \cdot S^{G}$-module, that is, $R \cdot S^{G}$ is a strongly separable $R$-algebra. Since $R \cdot S^{G}$ is a strongly separable $R$-algebra, the nilradical of $R$ lifts to the nilradical of $R \cdot S^{G}: \operatorname{nilrad}\left(R \cdot S^{G}\right)$ $=\operatorname{nilrad}(R) S^{G}$. Nilpotents map to nilpotents via ring automorphisms, thus $\operatorname{nilrad}(R)$ is a rational $G$-module. Hence, $\operatorname{nilrad}(R)=\operatorname{nilrad}\left(R^{G}\right)$ $\oplus(\operatorname{nilrad}(R))_{G}$, and $\operatorname{nilrad}\left(R \cdot S^{G}\right)=\operatorname{nilrad}\left(R^{G}\right) S^{G} \oplus(\operatorname{nilrad}(R))_{G} S^{G}$. The last equality follows since nilrad $\left(R^{G}\right) S^{G}$ is contained in $S^{G}$ and since $S_{G}$ is an $S^{G}$-module, $(\operatorname{nil} \operatorname{rad}(R))_{G} S^{G}$ is contained in $S_{G}$. Therefore, $\operatorname{nilrad}\left(S^{G}\right)$ $=\operatorname{nilrad}\left(R \cdot S^{G}\right) \cap S^{G}=\operatorname{nilrad}\left(R^{G}\right) S^{G}$.

Let $m$ be a maximal ideal in $R^{G}$ and consider the $G$-diagram

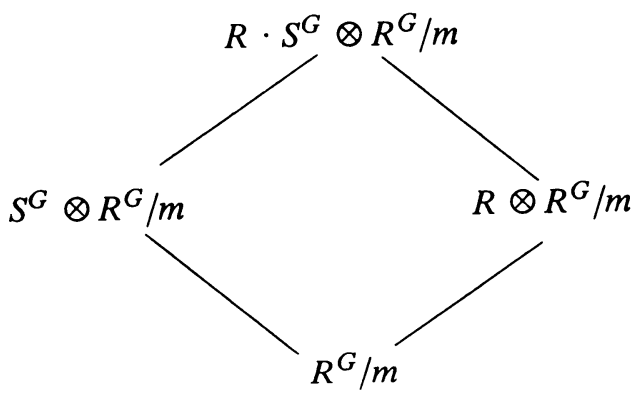

By the above $\operatorname{nilrad}\left(S^{G} \otimes R^{G} / m\right)=0$. Since $R \cdot S^{G} \otimes R^{G} / m$ is a finite $R$ module, by Lemma $3(\mathrm{~b}), S^{G} \otimes R^{G} / m$ is a finite $R^{G} / m$-module. Since $G$ is linearly reductive, $R^{G}$ is a finitely generated $k$-algebra; and hence, $R^{G} / m \simeq k$. This means we have $S^{G} \otimes R^{G} / m$ reduced and finite over the algebraically closed field $R^{G} / m$. Thus $S^{G} \otimes R^{G} / m$ is a separable $R^{G} / m$-algebra. With this happening for each maximal ideal in $R^{G}, S^{G}$ is a separable $R^{G}$-algebra [DI, 7.1, p. 72]. And by Lemma $3(\mathrm{~d}), S^{G}$ is a strongly separable $R^{G}$-algebra.

THEOREM 5. Suppose that we have a G-diagram of $S$ over $R$ in the Reductive Case and $S$ is a Galois extension of $R$ with group $\Gamma$ such that $\Gamma \mid S^{G}$ is contained in the ring automorphisms of $S^{G}$ and $\left(S^{G}\right)^{\Gamma}=R^{G}$. Then $S^{G}$ is a Galois extension of $R^{G}$ with group $\Gamma \mid S^{G}$ if and only if $R \cdot S^{G}$ is a Galois extension of $R$ with group $\Gamma \mid R \cdot S^{G}$.

Proof. If $S^{G}$ is a Galois extension of $R^{G}$, we apply Proposition 1(b).

Conversely, assume that $R \cdot S^{G}$ is a Galois extension of $R$ with group $\Gamma \mid R \cdot S^{G}$. Hence, $l: R \cdot S^{G} \otimes_{R} R \cdot S^{G} \rightarrow C\left(\Gamma \mid R \cdot S^{G}, R \cdot S^{G}\right)$ is an isomorphism. Since $\left(S^{G}\right)^{\Gamma}=R^{G}$, we need only show that $l^{\prime}: S^{G} \otimes_{R^{G}} S^{G} \rightarrow C\left(\Gamma \mid S^{G}\right.$, $\left.S^{G}\right)$ is surjective. Note that $C\left(\Gamma \mid S^{G}, S^{G}\right)$ is contained in $C\left(\Gamma \mid R \cdot S^{G}, R \cdot S^{G}\right)$. Let $f$ be in $C\left(\Gamma \mid S^{G}, S^{G}\right)$. Since $l$ is surjective, $f=l\left(\sum r_{i} s_{i} \otimes t_{i}\right)$ where we may assume $r_{i}$ in $R$ and $s_{i}, t_{i}$ in $S^{G}$. Hence, for each $\gamma$ in $\Gamma$, we have 


$$
f(\gamma)=l\left(\sum r_{i} s_{i} \otimes t_{i}\right)(\gamma)=\sum r_{i} s_{i} \gamma\left(t_{i}\right)=\sum r^{\prime} s_{i} \gamma\left(t_{i}\right)+\sum r_{i}^{\prime \prime} s_{i} \gamma\left(t_{i}\right)
$$

with $r_{i}^{\prime}+r_{i}^{\prime \prime}=r_{i}, r_{i}^{\prime}$ in $R^{G}, r^{\prime \prime}{ }_{i}$ in $R_{G}$. But $s_{i} \gamma\left(t_{i}\right)$ is in $S^{G}$, and by Lemma 2, $\sum r_{i}^{\prime} s_{i} \gamma\left(t_{i}\right)$ is in $S^{G}$ and $\sum r_{i}^{\prime \prime} s_{i} \gamma\left(t_{i}\right)$ is in $S_{G}$. But $f(\gamma)$ is in $S^{G}$. Hence, $\sum r_{i}^{\prime \prime} s_{i} \gamma\left(t_{i}\right)=0$ and $f(\gamma)=\sum r_{i}^{\prime} s_{i} \gamma\left(t_{i}\right)$. This means that

$$
f=l^{\prime}\left(\sum r_{i}^{\prime} s_{i} \otimes_{R^{G}} t_{i}\right)
$$

that is, $l^{\prime}$ is surjective.

We end the paper with an example in which $S$ is a Galois extension of $R$ and $S^{G}$ is a finite $R^{G}$-module, but $S^{G}$ is not a separable $R^{G}$-algebra.

EXAmPle 6. Assume $\operatorname{char}(k) \neq 2$. Let $m$ be even and relatively prime to $\operatorname{char}(k)$ and let $l_{1}$ and $l_{2}$ be positive integers such that $m l_{2}$ divides $l_{1}$. Let $R=k\left[x_{1}, x_{2}, 1 / x_{1}\right], S=k\left[x_{1}, x_{2}, 1 / x_{1}, x_{1}^{1 / m}\right], \Gamma=\left\{\gamma_{0}, \ldots, \gamma_{m-1} \mid \gamma_{i}\left(x_{1}^{1 / m}\right)\right.$ $\left.=\xi^{i} x_{1}^{1 / m}\right\}$ where $\xi$ is a primitive $m$ th root of unity, and $G=\mathrm{GL}_{1}(k)$ act on $S$ by $t\left(x_{1}\right)=t^{l_{1}} x_{1}$ and $t\left(x_{2}\right)=t^{l_{2}} x_{2}$ for $t$ in $G$. Note that $G$ is a linearly reductive algebraic group acting rationally on $S$ and $S$ is a Galois extension of $R$ with

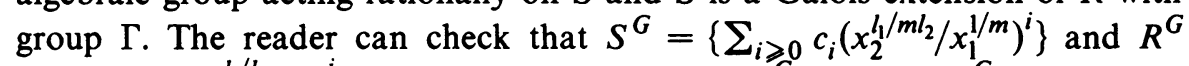
$=\left\{\sum_{i \geqslant 0} c_{i}\left(x_{2}^{l_{1} / l_{2}} / x_{1}\right)^{i}\right\}$. By Lemma $3(\mathrm{a})$, the ring $S^{G}$ is a finite $R^{G}$-module. Let $z=x_{2}^{1} / l_{2} / x_{1}$. Then we may think of $R^{G}=k[z]$ and $S^{G}=k\left[z, z^{1 / m}\right]$ $=R^{G}[w] /\left\langle w^{m}-z\right\rangle$. But $m$ is a unit in $S^{G}$ and $z$ is not a unit in $S^{G}$; hence, $S^{G}$ is not a separable $R^{G}$-algebra [J]. From Theorem 4 we know in this example that $S^{G}$ is not a separable $R^{G}$ algebra since $R \cdot S^{G}$ is not a separable $R$-algebra:

$$
R \cdot S^{G}=R[w]-\left\langle w^{m}-x_{2}^{l_{1} / l_{2}} / x_{1}\right\rangle .
$$

And $m$ is a unit in $R \cdot S^{G}$ but $x_{2}^{l_{1} / l_{2}} / x_{1}$ is not.

This paper is based on the author's doctoral dissertation (The University of Oklahoma, 1976). Thanks are due to Dr. Andy Magid under whose guidance this thesis was written.

\section{BIBLIOGRAPHY}

B N. Bourbaki, Commutative algebra, English transl., Hermann, Paris; Addison-Wesley, Reading, Mass., 1972. MR 50 \# 12997.

DI F. B. DeMeyer and E. Ingraham, Separable algebras over commutative rings, Lecture Notes in Math., vol. 181, Springer-Verlag, Berlin and New York, 1971. MR 43 \#6199.

F J. Fogarty, Invariant theory, Benjamin, New York, 1969. MR 43 \# 1458.

J G. J. Janusz, Separable algebras over commutative rings, Trans. Amer. Math. Soc. 122 (1966), 461-479. MR 35 \# 1585.

Air Force Institute of Technology/ENC Wright-Patterson AFB, Ohio 45433 